\title{
Towards a quantitative characterization of photoisomerization rates
}

\author{
Jürgen Troe \\ Institut fürPhysikalische Chemie, Universität Göttingen \\ Tammannstraße 6, D-37077 Göttingen, Germany
}

\begin{abstract}
Photoisomerization rates of energy-selected isolated molecules, of molecules in supercritical fluids and chusters, and of molecules in liquids at normal and elevated pressures can now be measured directly. The results can be analyzed by unimolecular rate theory, information of collisional energy transfer in gases and in compressed fluids, as well as knowledge on the solvent influence on the intramolecular motions, potentials and transport. By a combination of theory and empirical fitting of otherwise inaccessible quantities, a quantitative picture arises.
\end{abstract}

\section{INTRODUCTION}

Photoisomerization reactions in general are governed by a series of competing, sequential or parallel, photophysical processes. In order to arrive at a quantitative understanding, these processes have to be disentangled and characterized individually. The task appears comparably easy in the gas phase where the events can be separated. When higher densities are applied, in supercritical fluids, chusters, and liquids at ambient or elevated pressures, the separation becomes less well defined if not impossible. Modern experimental and theoretical techniques have opened new accesses towards a quantitative characterization of the phenomena involved. This report demonstrates the state of the art for a number of selected examples.

In low pressure gases, reactions on electronically excited potential energy surfaces often compete with fast internal conversions to such an extent that the main reaction occurs in the electronic ground state. In this case, the main reaction is intimately related to thermally activated reactions in the ground state. When the density is increased, the ground state component may become completely collisionally quenched such that the reactions in the electronically excited states dominate. These again may be influenced by collisional relaxation and other solvent effects. Whether the excited state reaction approaches a thermal unimolecular reaction in the electronically excited state or fast proceeds "downhill" towards ranges of electronically nonadiabatic dynamics, depends on the properties of the excited state potential energy surface and its modification by chustering or by reagent-solvent interaction. Besides "static" solvent effects, "dynamic" solvent effects of the viscosity-type may influence the dynamics in dense environments.

In the following, the photoisomerization reactions of cycloheptatrienes and of diphenyl polyenes serve as examples for the various aspects and the possibilities of a quantification.

\section{UNIMOLECULAR PROCESSES AND ENERGY RELAXATION IN EXCITED CYCLOHEPTATRIENES}

Light absorption in unsubstituted or substituted cycloheptatrienes in the near UV is known to induce a variety of parallel or sequential photoisomerization processes. In low pressure gases, about $97 \%$ of the excited molecules undergo fast internal conversion on the $100 \mathrm{fs}$ timescale into the electronic ground state where positional isomerizations via 1,5 hydrogen transfer and structural isomerizations to aromatic molecules (like toluene in unsubstituted cycloheptatriene) occur. The specific rate constants $k(E)$ for the structural isomerizations have been measured with pulsed laser techniques in real time [1]. Simulation of 
the results by "optimized" RRKM theory (i.e. fitting of one or two parameters, not more!) showed that thermal [2] and photoisomerization occur on the same path way, being different only in the distribution of vibrational energies of the reagents. The same probably applies to positional isomerization [3]. Examples for $k(E)$, which show well the predicted decrease of $k(E)$ with increasing extent of substitution, are shown in Fig. 1. Directly measured points and results from the analysis of Stern-Volmer plots of steady-state photoisomerization [4] (after calibration of collisional quenching through direct measurements of energy transfer [5]) fit very well to each other. Fig. 2 compares the specific rate constants for positional and structural isomerization. The picture well illustrates the different timescales of the two processes.

With increasing gas pressure, vibrationally excited cycloheptatriene molecules after internal converison are collisionally stabilized increasingly fast such that their isomerizations finally are completely suppressed. The collisional energy transfer process in the gas phase has been isolated and characterized quantitatively [5]. The process is now also theoretically understood completely by classical trajectory calculations [6],[7]. However, the complete simulation is fairly involved and requires adequate knowledge of the intermolecular potentials such that empirical tabulated information [8] often proves to be useful. With the described information, Stern-Volmer plots of structural and positional photoisomerizations of cycloheptatrienes can be understood quantitatively [1].

With further increasing density of the medium, the appearance of the photoisomerization changes: a valence tautomerization to bicyclo[3,2,0]hepta-2,6-diene $(\mathrm{BCH})$ and positional isomerizations involving 1,7 hydrogen shifts appear [9]. In medium pressure gases, also benzene and (probably) $\mathrm{CH}_{2}$-related products are formed [10],[11]. The question how are the gas phase and the liquid phase photoisomerizations connected, appears in a new light following recent measurements of the rate of collisional quenching of excited cycloheptatriene in compressed supercritical fluids and liquid solvents [12]. It was shown that the rate of this process increases proportional to the bath gas pressure up to about 50 bar before a slower increase sets in, see Fig. 3. Nevertheless, the rate of quenching in normal liquids is so high that not only structural but also positional isomerization of ground state cycloheptatriene is completely suppressed in liquids. The chemical changes observed in the liquid phase, therefore, must be unrelated to processes following internal conversion.

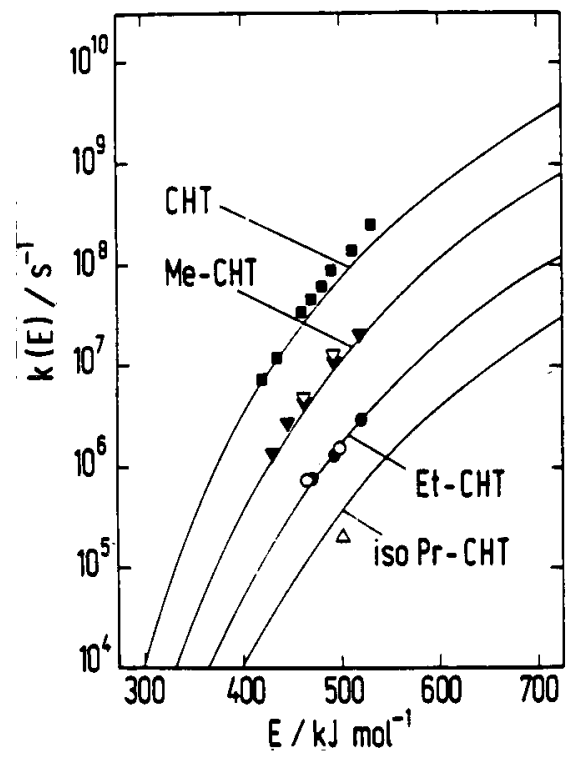

Fig. 1 Specific rate constants $k(E)$ of the unimolecular isomerizations of alkyl cycloheptatrienes (CHT) [1]. (Open symbols: from time-resolved measurements, filled symbols: from Stern-Volmer plots of isomerization quenching and direct measurements of energy transfer, full line: RRKM calculations fitted to data for thermal isomerization of CHT).

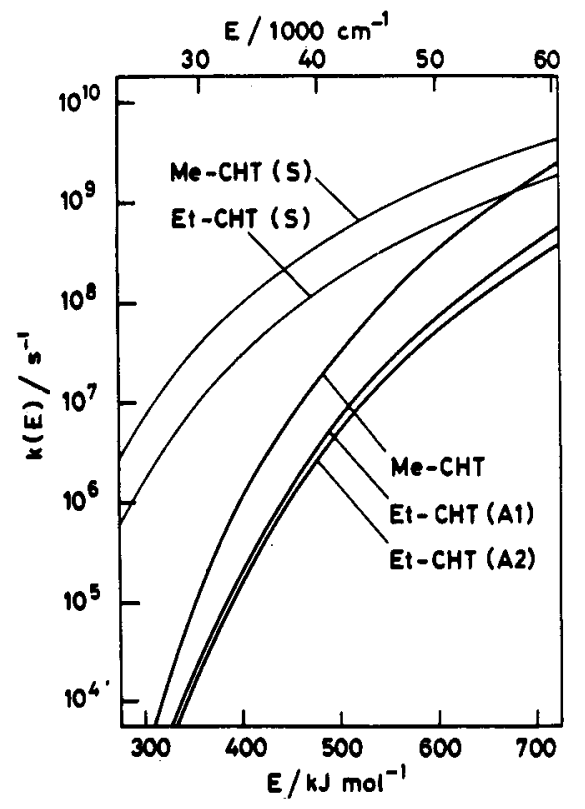

Fig. 2 Specific rate constants $k(E)$ for positional isomerization of alkyl cycloheptatrienes (S) and for aromatization (A1, A2) [4]. (RRKM calculations fitted to data for thermal isomerizations from [1] and [3]). 


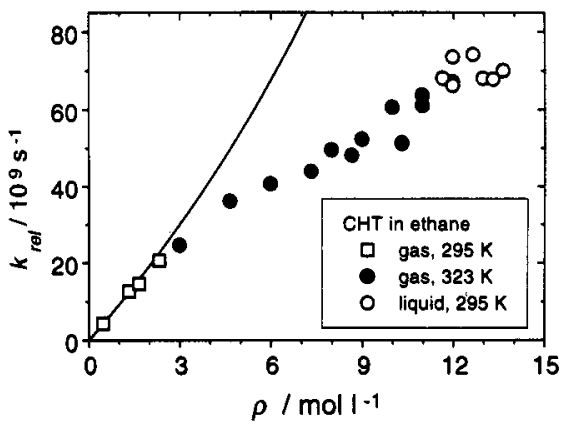

Fig. 3 Rate constants for collisional deactivation of vibrationally highly excited cycloheptatriene in compressed ethane [12].

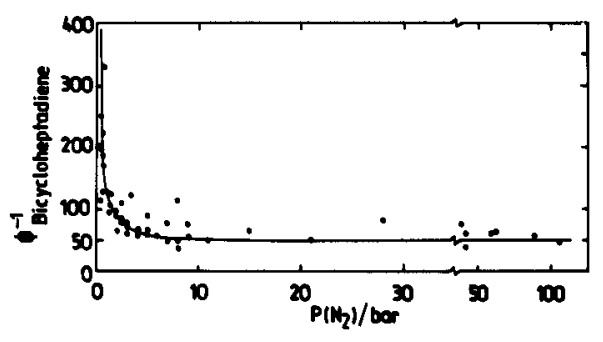

Fig. 4 Bicycloheptadiene yield in the photoisomerization of cycloheptatriene in compressed $\mathrm{N}_{2}$ [11].

More information on the pathway towards the bicyclic $\mathrm{BCH}$ was derived by measuring $\mathrm{BCH}$ and benzene quantum yields in compressed gases [11]. It was shown that benzene is the low pressure product and that it needs a few bar pressure (half quenching pressure of about 2 bar of $\mathrm{N}_{2}$ ) to stabilize $\mathrm{BCH}$, see Figs. 4 and 5. This indicates that, also in the excited electronic state there is competition between collisional quenching and unimolecular transformation of electronically and vibrationally excited species. Nevertheless, the formtion of benzene is not completely suppressed at high pressures which may indicate the presence of a thermal dissociation of the $\mathrm{BCH}$ precursor in the excited electronic state. Whether, after collisional quenching in the excited electronic state, thermal tautomerization in this state towards $\mathrm{BCH}$ and positional isomerizations compete, remains to be investigated in further experiments using gas pressures above 10 bar. At least we know that, in principle, one can quantitatively characterize the contributing processes such as collisional energy transfer in dense fluids and unimolecular rearrangements describable by RRKM theory.

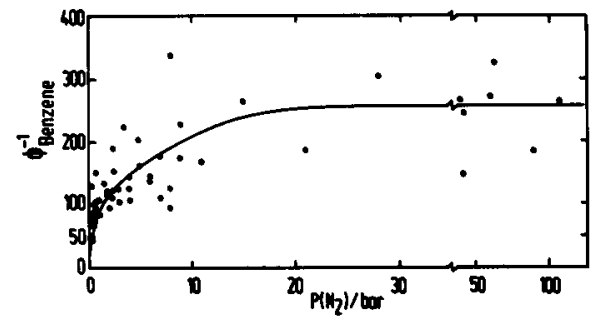

Fig. 5 Benzene yield in the photoisomerization of cycloheptatriene in compressed $\mathrm{N}_{2}$ [11].

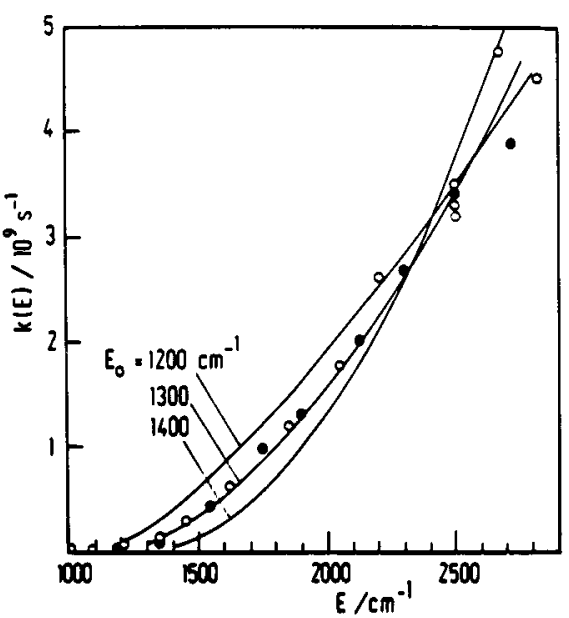

Fig. 6 Specific rate constants $\mathrm{k}(\mathrm{E})$ for the isomerization of electronically excited trans-stilbene ( 0 : experiments from [15], ๑: experiments from [16], full lines: RRKM fits with variable threshold energies $E_{o}$ from [21]).

\section{UNIMOLECULAR PROCESSES OF ISOLATED EXCITED DIPHENYLPOLYENES}

Diphenylpolyenes are particulary well suited to studied the elementary steps of photoisomerization processes. The general situation is quite comparable to the cycloheptatrienes: electronic excitation followed by internal conversion opens an access to vibrationally highly excited molecules in the electronic ground state, allowing to study isomerization and energy transfer of "hot" ground state molecules. While the study of collisional energy transfer of highly excited electronic ground state trans-stilbene has been 


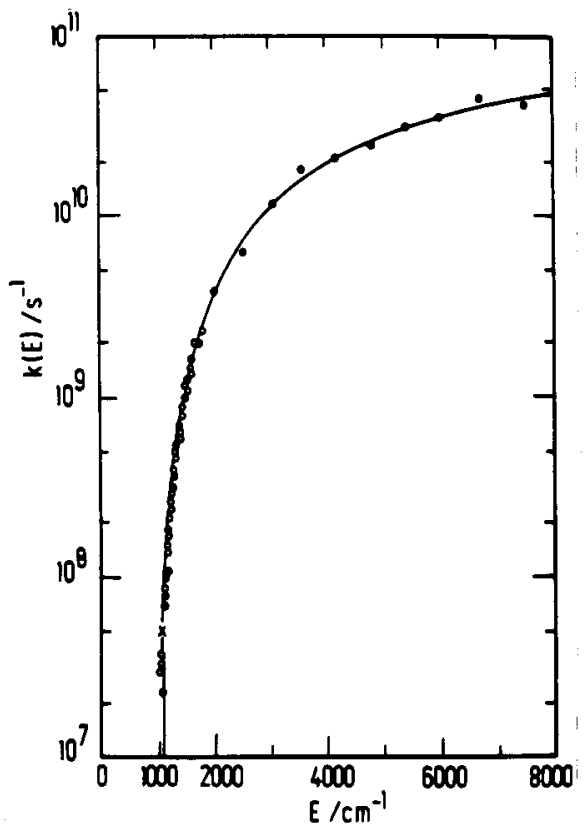

Fig. 7 Specific rate constants $k(E)$ for the isomerization of electronically excited diphenyl butadiene (o: experiments from [18], $\bullet$ : experiments and full line: RRKM fit to the experiments from [19]).

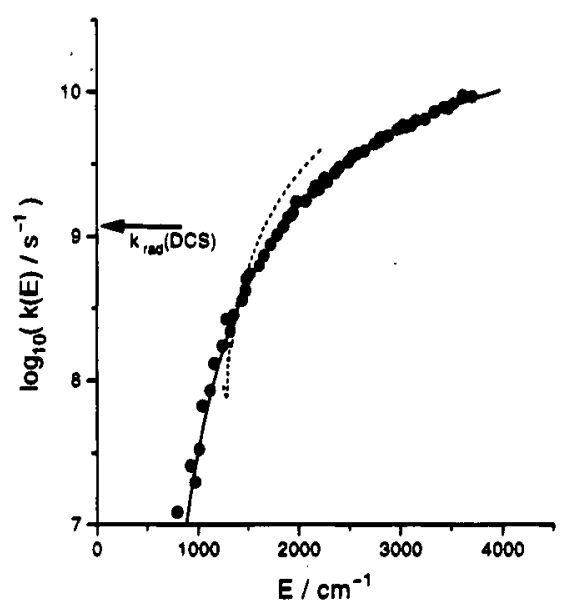

Fig. 8 Specific rate constants $k(E)$ for the isomerization of electronically excited dimethylamino cyanostilbene $(\bullet)[20]$ and trans-stilbene [17] (dashed line) (full and dashed lines: RRKM fits to the experiments).

investigated exciting cis-stilbene as a precursor [13],[14], unimolecular processes of electronic ground state molecules so far have not been monitored in detail. However, specific rate constants $\mathrm{k}(\mathrm{E})$ of isomerization of jet-cooled isolated trans-stilbene [15]-[17], diphenylbutadiene [18],[19], and 4dimethylamino-4'-cyanostilbene [20] in their excited electronic states were measured over wide ranges of $\mathrm{k}(\mathrm{E})$. In all cases, an optimized RRKM representation (fit of two parameters only) was in perfect agreement with the experiments [19],[21]. Figs. $6-8$ demonstrate the general behavior. The fit is never unique, several sets of activated complex frequencies achieving nearly the same task [21]. Contrary to the optimism of some quantum chemists, activated complex properties in these large systems and in their electronically excited states cannot yet be calculated with sufficient precision. Also, anharmonicity effects in the density of states $\rho(E)$ contained in $k(E)$ cannot yet be established with confidence [22]. Therefore, the fitting procedure at present remains the method of choice. However, the high precision of the measurements allows us to investigate some basic questions: e.g. one may ask whether the K-quantum number of rotating molecules is strictly conserved during the isomerization or looses its significance. In the latter case, one might see threshold rate constants markedly below $k\left(E=E_{\circ}\right)=1 / h \rho(E)$ [19].

\section{PHOTOISOMERIZATION OF DIPHENYLPOLYENES IN CLUSTERS AND IN COMPRESSED FLUIDS}

If there were no reagent-solvent interactions apart from rapid energy exchange in collisions, thermal averaging of specific rate constants $k(E)$ should lead to the high pressure or liquid photoisomerization rates of electronically excited diphenylpolyenes. In reality, however, large differences are observed (acceleration by a factor of 50 in trans-stilbene, a factor of 2 in diphenylbutadiene). Fig. 9 shows the predicted pressure dependence (falloff curve of the thermal unimolecular isomerization combined with a transition to the Kramers-Smoluchowski high damping range) for trans-stilbene photoisomerization in comparison to measurements [23]. In the case with the most pronounced differences between observed and modeled (without specific solute-solvent interaction) curves, i.e. in trans-stilbene, the differences occur surprisingly 


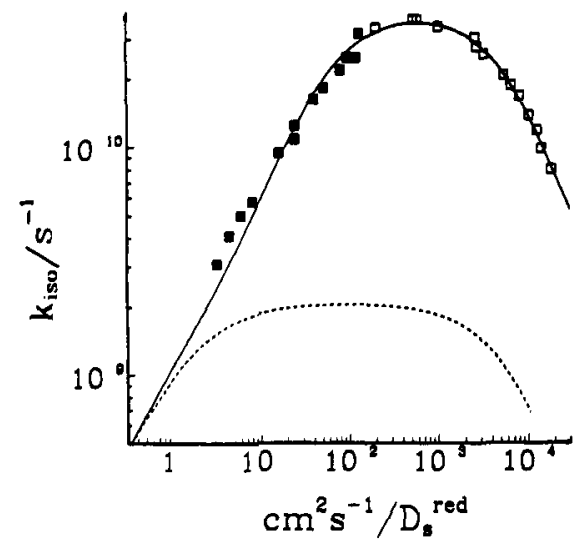

Fig. 9 Thermal rate constants for isomerization of electronically excited trans-stilbene in compressed methane ( $\square$ ) and ethane ( $\square$ ) as a function of the inverse self-diffusion coefficient $D_{s}^{\text {red }}$ of the bath gases [23] (dashed curve: RRKM-Kramers/

Smoluchowski model based on gas phase experiments, see fig. 6, full curve: as dashed curve but with solvent modification of the barrier).

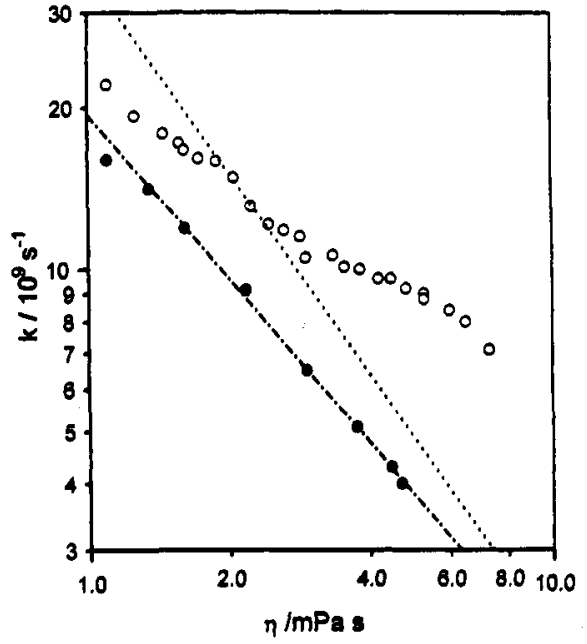

Fig. 10 Thermal rate constants for isomerization of electronically excited trans-stifbene (0) and diphenyl butadiene ( $\bullet$ ) in compressed liquid ethanol [24] (dashed line: dielectric relaxation of ethanol).

early in only moderately compressed gases. This indicates that strong modifications of the potential relevant for excited state photoisomerization already occur in the first reagent-solvent clusters being formed. After this "solvent shift" of the photoisomerization barrier, in the considered case apparently no more pressure effects occur which contribute to what chemists like to call an "activation volume". The decrease of the photoisomerization rate at very high solvent pressures leads to a rate which is imversely proportional to the solvent viscosity. Obviously, this corresponds to the conventional high damping limit of the Kramers-Smoluchowski treatment of transport-controlled processes. In this case, the reaction flux over the reaction energy barrier is hindered by the solvent environment in a viscosity-type way. The bulk viscosity of the solvent apparently provides the magnitude of this friction effect. Evidently, this pressure effect on the reaction flux should not be included in an interpretation of the "activation volume". The described solvent effects are similar in character in the photoisomerization of diphenyl butadiene; however, the differences between observed and measured pressure dependences are much smaller than in transstilbene (only a factor of 2 instead of 50). Apart from the dynamic solvent effects of the KramersSmoluchowski type described above, other interesting dynamic solvent effects have been observed in transstilbene, i.e. in a system with particularly "labile" photoisomerization energy barrier [24]. The pressure dependence of the photoisomerization rate in compressed alcohols shows a kink-type form, see fig. 10, in contrast to diphenylbutadiene. Apparently, solvent dielectric relaxation here competes with chemical

reaction. At low pressures, dielectric relaxation is so fast that the reaction barrier is lowered by the new orientation of the solvent shell; at high pressures, the overcoming of the barrier is faster than the dielectric relaxation such that the reaction occurs over a higher barrier corresponding to the unrelaxed orientation of the solvent shell. Only in trans-stilbene, with its high sensitivity of the barrier to the solute-solvent "static" interaction and with different "dynamic" viscosity dependences of barrier crossing and dielectric relaxation, this effect so far has been observed. By comparing these results with those for diphenylbutadiene (Fig. 10) it can be seen that the butadiene exhibits no such effects.

Surprisingly, marked effects of solvent pressure, which can be separated into "static" and "dynamic" solvent effects, have also been found in the "ultrafast downhill" photoisomerization of electronically excited cis-stilbene [25]. First, the downhill motion again is attenuated by viscosity-type solute-solvent interactions. In addition, however, a modification of the slope of the potential by the particular solvents becomes quite apparent. Solvent effects of this type wait for a theoretical explanation on the basis of more advanced quantum-chemical calculations of solute-solvent interactions. 


\section{REFERENCES}

1. H. Hippler, K. Luther, J. Troe, and H. J. Wendelken. J.Chem. Phys. 79, 239 (1983).

2. D. C. Astholz, J. Troe, and W. Wieters. J. Chem. Phys. 70, 5107 (1979).

3. K. Egger. J. Amer. Chem. Soc. 89, 3688 (1967); 90, 1 (1968); 90, 6 (1968).

4. J. Troe and W. Wieters. J.Chem. Phys. 71, 3931 (1979).

5. H. Hippler, J. Troe, and H. J. Wendelken. J. Chem. Phys. 78, 6718 (1983).

6. Th. Lenzer, K. Luther, J. Troe, R. G. Gilbert, and K. F. Lim J. Chem. Phys. 103, 626 (1995).

7. Th. Lenzer and K. Luther. J. Chem. Phys. 104, 3391 (1996).

8. H. Hippler and J. Troe. In Bimolecular Collisions (eds. J. E. Baggott and M. N. R. Ashfold, The Royal Society of Chemistry, London 1989).

9 A. P. ter Borg, E. Razenberg, and H. Kloosterziel. Chem. Comm. 1210 (1967).

10. R. Srinivasan. J. Amer. Chem. Soc. 84, 3432, 4141 (1962); Adv. in Photochem. 4, 113 (1966).

11. H. Hippler, L. Schubert, and J. Troe. Unpublished results.

12. J. Benzler, S. Linkersdörfer, and K. Luther. J. Chem. Phys., submitted, 1996.

13. R. J. Sension, S. T. Repinec, and R M. Hochstrasser. J. Chem. Phys. 98, 6291 (1993);

H. Petek, Y. Fujiwara, D. Kim, and K. Yoshihara. J. Amer. Chem. Soc. 110, 6269 (1988).

14. L. Nikowa, J. Schroeder, D. Schwarzer, and J. Troe. Ber. Bunsenges. Phys. Chem. 98, 262 (1994); L. Nikowa, D. Schwarzer, and J. Troe. Chem. Phys. Lett. 233, 303 (1995).

15. J. Syage, W. R. Lambert, P. M. Felker, A. H. Zewail, and R. M. Hochstrasser. Chem. Phys. Lett. 88, 266 (1982).

16. A. Amirav and J. Jortner. Chem. Phys. Lett. 95, 295 (1983).

17. Ch. Lienau, J. Schroeder, and J. Troe. Unpublished Results.

18. J. F. Shepanski, B. W. Keelan, and A. H. Zewail. Chem. Phys. Lett. 103, 9 (1983).

19. J. Troe, A. Amirav, and J. Jortner. Chem. Phys. Lett. 115, 245 (1985).

20. R. Daum, T. Hansson, R. Nörenberg, D. Schwarzer, and J. Schroeder. Chem. Phys. Lett. 246, 607 (1995).

21. J. Troe. Chem. Phys. Lett. 114, 241 (1985).

22. J. Troe. Chem. Phys. 190, 381 (1995).

23. J. Schroeder and J. Troe. In Activated Barrier Crossing (eds. G. R. Fleming and P. Hänggi, World Scientific, Singapore 1993) p. 206.

24. R. Mohrschladt, J. Schroeder, D. Schwarzer, J. Troe, and P. Vöhringer. J. Chem. Phys. 101, 7566 (1994).

25. L. Nikowa, D. Schwarzer, J. Troe, and J. Schroeder. J. Chem. Phys. 97, 4827 (1992). 